\title{
Neurons light the way
}

Monitoring the activity of neurons in vivo in the freely behaving zebrafish larvae is now possible using bioluminescence, an approach with great potential for unveiling how neuronal networks control behavior.

Understanding how the activity of specific sets of neurons in the brain drives particular actions requires monitoring neuronal activity while the animals are free to move. Neural recordings in freely behaving animals are possible with electrophysiology, but this technique is restricted to animals that can transport the bulky electronic devices. Alternatively, in vivo fluorescence imaging allows noninvasive monitoring of neuronal activity in awake animals but requires them to be restrained, paralyzed or anesthetized.

Florian Engert at Harvard University and his collaborators now use a nonimaging approach that exploits bioluminescence to study how brains process sensory information and elicit behaviors in the larvae zebrafish. The translucent body of the fish larvae allows monitoring each individual neuron in its brain, but until now it was not possible to record the activity of these neurons while the fish is freely swimming around.

Engert's group created transgenic zebrafish in which a fusion of the jellyfish bioluminescent protein Aequorin and GFP was expressed in specific sets of neurons. This fusion protein responds to changes in neuronal calcium-ion levels by emitting green photons without producing background light emission or requiring excitation light.

By placing a large-area photodetector directly above the fish tank and simultaneously recording fish movements using an infrared camera, the researchers detected large and fast bioluminescent signals that were related to neural activity in the freely moving zebrafish larvae. This setup enabled continuous and long-term monitoring of the 'neuroluminescence' with high temporal resolution, albeit at the expense of all spatial information.

This lack of spatial information can be indirectly regained, however, by specifically targeting the expression of the reporter to neurons of interest. Using this strategy,
Engert and colleagues genetically targeted GFP-Aequorin expression to neurons of the hypocretin/orexin hypothalamic system that control arousal in mammals and fish. They detected bioluminescence from this group of merely 20 neurons, and the signal was associated with periods of increased locomotor activity. Moreover, the technique had enough resolution to detect signals from a single neuron and to distinguish two classes of neural activity associated with different swimming behaviors.

Not satisfied with the fact that the setting was limited to studying fish behavior in the dark, Engert and collaborators designed a detection system that allows illuminating the setting with fast flickering visible light while at the same time recording the bioluminescence signal. This extends the utility of this technique to the investigation of visually driven behaviors.

As well as performing simple experiments to compare neuronal activity in freely moving and tethered fish, Engert's group intends to use this technology to study specific neuronal networks during hunting and prey-capture behavior. "Zebrafish are very visual animals; we will allow them to see and hunt by vision while monitoring the activity of genetically targeted dopaminergic and serotonergic neurons," explains Engert.

Currently, the system is limited to the detection of bursts of a few spikes and lacks the sensitivity to detect individual action potentials. But Engert is hopeful that generation of modified forms of GFP-Aequorin that increase its calcium sensitivity as well as that development of improved light detectors and photoncounting imaging setups will expand the possibilities of the nonimaging field. This technique also holds great potential in its application to other neuroscience models such as Drosophila melanogaster larvae or Caenorhabditis elegans, by simply following the neurons' luminous ways.

\section{Erika Pastrana}

\section{RESEARCH PAPERS}

Naumann, E. A. et al. Monitoring neural activity with bioluminescence during natural behavior. Nat. Neurosci. 13, 513-520 (2010). 